\title{
Estudio Descriptivo del Sistema Hospitalario en Relación a Pacientes Sometidos a Tratamiento Quirúrgico por Trauma Facial en el Hospital Hernán Henríquez Aravena de Temuco, Chile
}

\author{
Descriptive Study of Hospital System in Relation to Patients Undergoing Surgical \\ Treatment of Facial Trauma in the Hernan Henriquez Aravena Hospital of Temuco, Chile
}

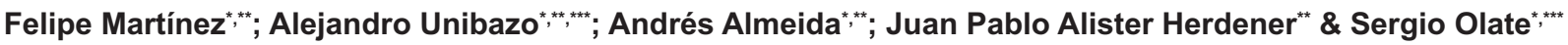

MARTÍNEZ, F.; UNIBAZO, A.; ALMEIDA, A.; ALISTER, H. J. P. \& OLATE, S. Estudio descriptivo del sistema hospitalario en relación a pacientes sometidos a tratamiento quirúrgico por trauma facial en el Hospital Hernán Henríquez Aravena de Temuco, Chile. Int. J. Odontostomat., 5(2):141-146, 2011.

RESUMEN: El trauma maxilofacial conforma una serie de lesiones que involucran tejidos óseos y blandos, provocando alteraciones funcionales y estéticas. El objetivo de este trabajo es determinar el perfil de pacientes que son sometidos a tratamiento quirúrgico por trauma maxilofacial en los pabellones del Hospital Regional de Temuco. De la base de datos del centro de estadísticas del Hospital Regional Hernán Henríquez Aravena, se obtuvieron un total de 145 sujetos con trauma maxilofacial entre los años 2008 y 2009. De la misma base de datos se obtuvieron los datos con los que se determinó la frecuencia según año, etnia, sexo, edad, sistema de previsión, mes, jornada, servicio, diagnóstico de fractura, manejo de tejidos blandos y tratamiento. En este estudio se encontraron 28 casos no diagnosticados (19,3\%), 35 fracturas de cigomático $(24,1 \%), 17$ fracturas de piso de orbita $(11,7 \%), 22$ fracturas de otros huesos del cráneo $(15,2 \%), 21$ fracturas de mandíbula $(14,5 \%), 11$ fracturas múltiples faciales $(7,6 \%), 3$ fracturas de base de cráneo $(2,1 \%)$ y 8 otros $(5,5 \%)$. Como conclusión podemos decir que las fracturas de cigomático es la más prevalente. Respecto al tratamiento más realizado en el trauma es la reducción con osteosíntesis múltiple.

PALABRAS CLAVE: tratamiento quirúrgico, trauma maxilofacial, fracturas faciales.

\section{INTRODUCCIÓN}

El trauma es la tercera causa de muerte en población chilena (Rojas et al., 2002), de forma que el trauma facial un motivo importante para la consulta hospitalaria.

La variabilidad de presentaciones clínicas, la diversidad de etiología y las diferencias en los tipos de tratamientos limita la elaboración y ejecución de protocolos clínicos que sean útiles a todos los servicios de urgencia (Le et al., 2003). Junto a ello, la diversa formación clínica de los profesionales responsables del manejo de estos pacientes y las características poblacionales de los lugares donde ocurren, hacen que el tratamiento del trauma facial sea complejo.

De esta forma, los elementos estadísticos y epidemiológicos sobre las fracturas faciales son muy importantes tanto para el control eficaz del tratamiento como para el desarrollo de medidas de prevenciones útiles y aplicadas a la localidad estudiada (Lee et al., 2010).

El objetivo de esta investigación es realizar un análisis de los pacientes sometidos a tratamiento qui-

\footnotetext{
* Unidad de Cirugía Oral y Maxilofacial, Hospital Hernán Henríquez Aravena, Temuco, Chile.

** Unidad de Cirugía Oral y Maxilofacial, Facultad Odontología, Universidad Mayor, Temuco, Chile.

*** Unidad de Cirugía Oral y Maxilofacial, Facultad de Medicina, Universidad de La Frontera, Temuco, Chile.
} 
MARTíNEZ, F.; UNIBAZO, A.; ALMEIDA, A.; ALISTER, H. J. P. \& OLATE, S. Estudio descriptivo del sistema hospitalario en relación a pacientes sometidos a tratamiento quirúrgico por trauma facial en el Hospital Hernán Henríquez Aravena de Temuco, Chile. Int. J. Odontostomat., 5(2):141-146, 2011.

rúrgico para el manejo de las fracturas faciales en el Hospital Hernán Henríquez Aravena (HHHA) de Temuco, Chile en el periodo $2008-2009$.

\section{MATERIAL Y MÉTODO}

Fue diseñado un estudio descriptivo, analizando la totalidad de los pacientes con diagnóstico de trauma facial tratados de forma quirúrgica en el HHHA entre los años 2008 y 2009.

La información necesaria se obtuvo a partir de la base de datos perteneciente al servicio de estadísticas del HHHA, para lo cual fueron aprobados los permisos necesarios por parte del centro hospitalario; los criterios de inclusión se remitieron al año de búsqueda (2008 ó 2009) y al hecho de ser realizado el tratamiento quirúrgico en el paciente; cualquier deficiencia en la ficha clínica fue incorporado dentro de los elementos de estudio.

Se diseñó una ficha clínica especialmente para estudiar las variables, que fueron: año de la intervención, etnia, sexo, edad, seguro de salud, mes de ocurrencia, jornada utilizada para realizar la cirugía, servicio quirúrgico responsable del procedimiento, diagnóstico de la fractura, tratamiento realizado, entre otros.

El ingreso de los datos se realizó en una planilla Excel (Microsoft Office 2007®) y el análisis de datos fue realizado por medio del programa SPSS 15 (Stata®). Se realizó análisis descriptivo de los datos, agrupándolos en tablas de distribución de frecuencias y gráficos.

\section{RESULTADOS}

De 21.488 pacientes sometidos a diagnóstico y tratamiento en el HHHA de Temuco, 145 presentaron trauma facial de abordaje quirúrgico, representando un $4 \%$ del trauma total presente en el $\mathrm{HHHA}$.

En cuanto al sexo, los hombres siempre sufrieron mayor cantidad de fracturas tratadas de forma quirúrgica en comparación a las mujeres, correspondiendo a un $91,7 \%$ de los fracturados al sexo masculino (Tabla I). En términos de edad, se observó también que la mayor cantidad de pacientes tratados tenían entra los 20 y 29 años de edad con un 29,7\% (Fig. 1).

Los aspectos económicos evaluados en este estudio, señalaron que los pacientes tratados de forma quirúrgica en el HHHA correspondían al segmento

Tabla I. Distribución del total de pacientes sometidos a trauma maxilofacial tratados en el Hospital Regional HHA, Temuco, Chile, según sexo.

\begin{tabular}{lcc}
\hline Sexo & $\mathbf{n}$ & $\%$ \\
\hline Mujeres & 12 & 8,3 \\
Hombres & 133 & 91,7 \\
\hline Total & 145 & 100,0 \\
\hline
\end{tabular}

Tabla II. Distribución del total de pacientes sometidos a trauma maxilofacial tratados en el Hospital Regional HHA, Temuco, Chile, según jornadas.

\begin{tabular}{lcc}
\hline Jornadas & $\mathbf{n}$ & $\%$ \\
\hline Mañana & 87 & 60,0 \\
Tarde & 58 & 40,0 \\
\hline Total & 145 & 100,0 \\
\hline
\end{tabular}

\section{EDADES}

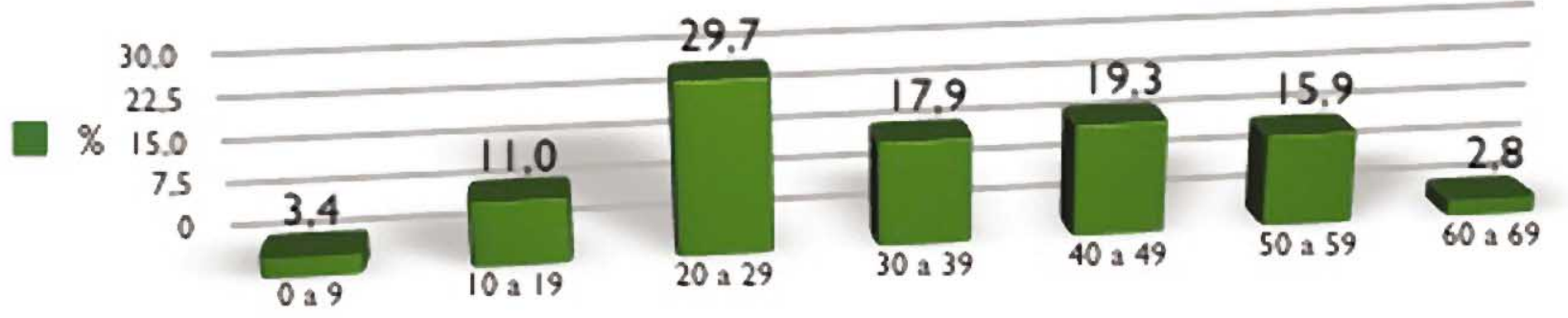

Fig. 1. Porcentajes de pacientes sometidos a cirugía debido a la presencia de trauma maxilofacial en el Hospital HHA, según la edad de los 144 pacientes. 
MARTíNEZ, F.; UNIBAZO, A.; ALMEIDA, A.; ALISTER, H. J. P. \& OLATE, S. Estudio descriptivo del sistema hospitalario en relación a pacientes sometidos a tratamiento quirúrgico por trauma facial en el Hospital Hernán Henríquez Aravena de Temuco, Chile. Int. J. Odontostomat., 5(2):141-146, 2011.

de FONASA A en un $53,8 \%$, seguido de pacientes del sector privado en un $22,8 \%$ de los casos (Fig. 2). También asociado al costo del tratamiento, el más prevalente fue la reducción con osteosíntesis múltiple con $75,2 \%$ (Fig. 4).

El $42,1 \%$ de los casos fueron tratados en el año 2008 y el $57,9 \%$ en el año 2009 . Se estableció que el mayor número de procedimientos quirúrgicos fue realizado en el mes de enero con un $12,4 \%$ sufriendo una brusca disminución para el mes de febrero con un 4,8\% (Fig. 3). En términos de planificación y desarrollo quirúrgico, la jornada de la mañana fue la más demandada con un $60 \%$ del total de los procedimientos (Tabla II).
Tabla III. Distribución del traumatismo maxilofacial, según diagnóstico.

\begin{tabular}{lcc}
\hline Dagnósticos & $\mathbf{n}$ & $\mathbf{\%}$ \\
\hline No Diagnosticado & 28 & 19,3 \\
Fractura de hueso cigomático & 35 & 24,1 \\
Fractura de piso de la órbita & 17 & 11,7 \\
Fractura de otros huesos del cráneo & 22 & 15,2 \\
Fractura mandibular & 21 & 14,5 \\
Fracturas múltiples faciales & 11 & 7,6 \\
Fractura de base de cráneo & 3 & 2,1 \\
Otros & 8 & 5,5 \\
\hline Total & 145 & 100,0 \\
\hline
\end{tabular}

\section{SISTEMA DE PREVISION}

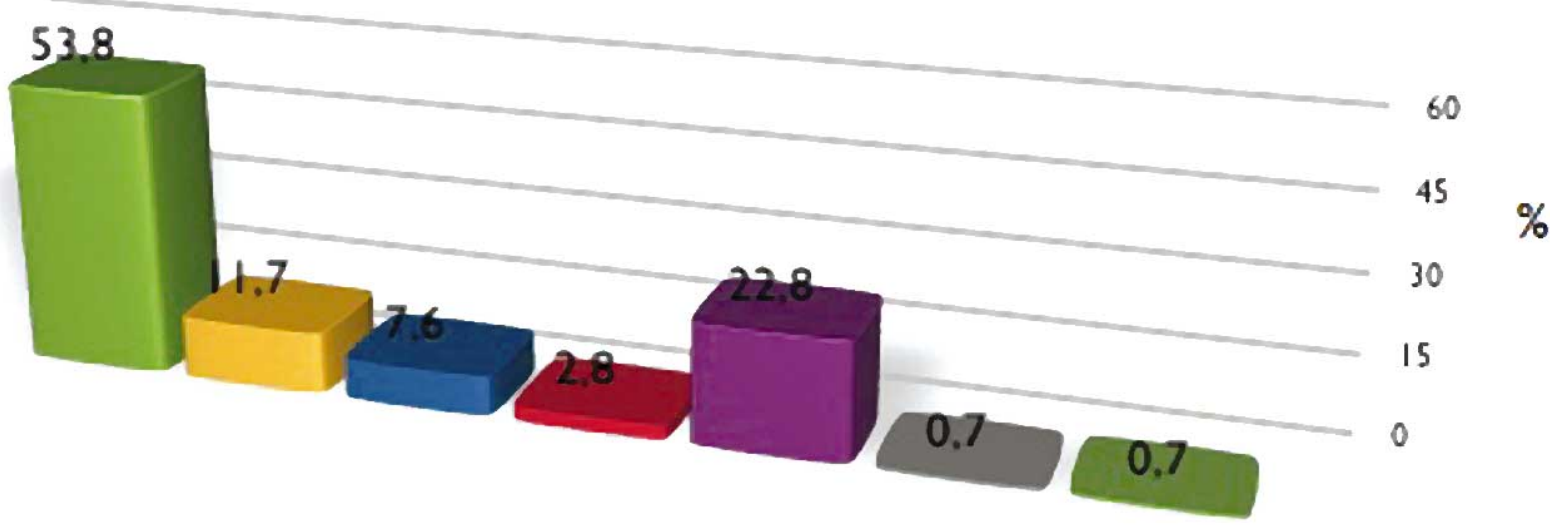
FONASA A
FONASA B
FONASA C
PRAIS
SEGURO ESCOLAR
FONASA D
PRIVADOS

\section{PRAIS}

Fig. 2. Porcentajes de pacientes sometidos a cirugía debido a la presencia de trauma maxilofacial en el Hospital HHA, agrupados según sistema de previsión.

\section{TRATAMIENTO}

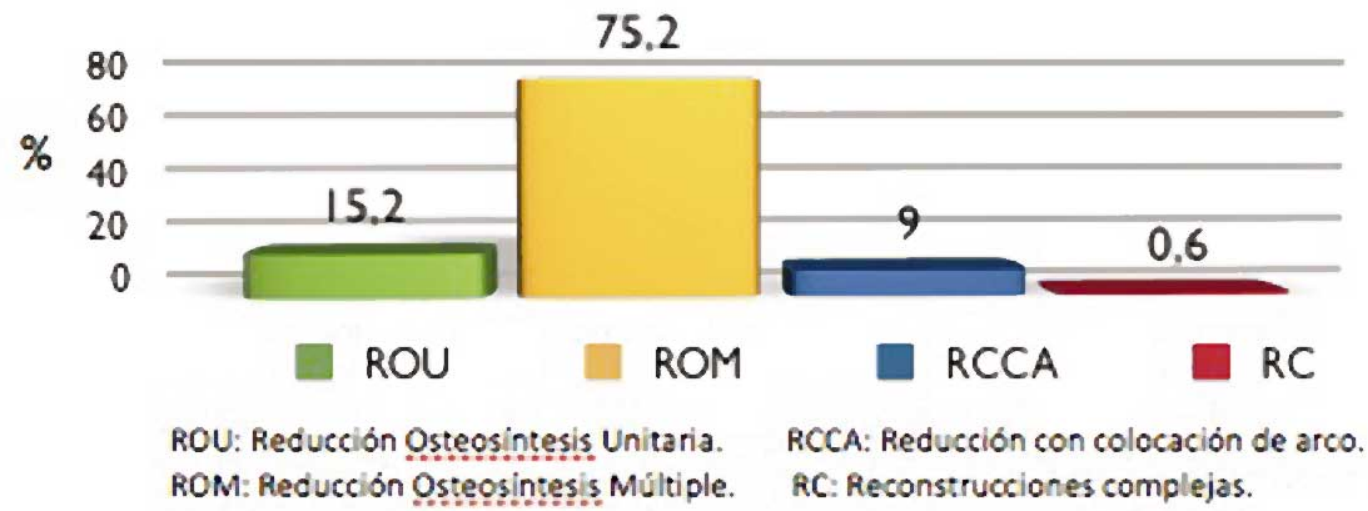

Fig. 3. Porcentajes de tratamientos de los 144 pacientes operados por trauma maxilofacial en el Hospital HHA. (ROU: Reducción Osteosíntesis Unitaria, ROM: Reducción Osteosíntesis Múltiple, RCCA: Reducción con colocación de arco, RC: Reconstrucciones complejas). 


\section{MES}

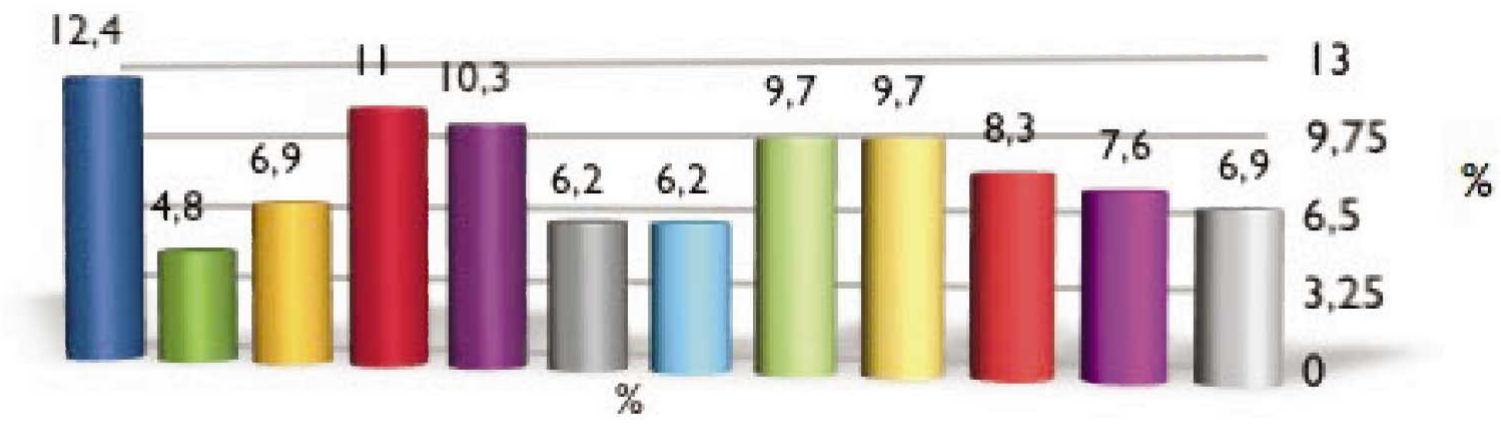

$\begin{array}{lllll}\text { ENERO } & \text { FEBRERO } & \text { MARZO } & \text { ABRIL } & \text { MAYO } \\ \text { JULIO } & \text { AGOSTO } & \text { SEPTIEMBRE } & \text { OCTUBRE }\end{array}$

Fig. 4. Distribución de las atenciones quirúrgicas de los 144 pacientes durante los dos años de evaluación según el mes en que se trató.

En el caso de los diagnósticos realizados, se observó que el $19,3 \%$ de los documentos estudiados no presentaba descripción del diagnóstico; por otra parte, si se pudo constatar que las fracturas de hueso cigomático $(24,1 \%)$ y las de mandíbula $(14,5 \%)$ fueron las más frecuentemente tratadas (Tabla III).

\section{DISCUSIÓN}

Los resultados de esta investigación permiten entender los protocolos de trabajo en centros hospitalarios de alta complejidad; en nuestro estudio, el mes con mayor actividad fue enero $(12,4 \%)$ y el menor febrero (4,8\%); sin embargo, la estación del año donde más se operó el trauma fue en otoño $(28,3 \%)$, estos resultados son distintos a los de Erol et al. (2004), quienes en su estudio retrospectivo de 25 años mencionan que la mayoría de los traumas maxilofaciales ocurrieron en verano. Nuestra región aumenta la población flotante en temporada estival, debido a que el turismo es una actividad económica habitual, lo que puede justificar un alto nivel de abordajes quirúrgico en el mes de enero (mayor demanda); por otra parte, las vacaciones del personal público son generalmente en febrero, lo que también puede explicar la abrupta disminución de los procedimientos durante este mes.

La jornada con más demanda fue la mañana con un $60 \%$ de las atenciones. En el HHHA existe un departamento clínico - quirúrgico que atiende derivaciones del servicio de urgencia y de hospitales de menor complejidad durante el dia pues no existe cirujano maxilofacial de urgencia o llamado; de esta forma, las intervenciones quirúrgicas son generalmente durante el periodo de la mañana y no durante la noche o la tarde ya que el servicio no se encuentra operativo en tales periodos.

En nuestros resultados, el 19,3\% de los casos no presentó diagnóstico. Medina et al. (2006) en un estudio realizado en el mismo recinto asistencial también reportaron la deficiencia en esta etapa, demostrando la persistencia de esta falencia. Esta información es un elemento importante para planificar las actividades de un servicio quirúrgico, de forma que esta conducta, mantenida en el tiempo, puede ser en parte responsable de algunas deficiencias en la admisión y en la administración del servicio.

Un total de 145 pacientes con trauma maxilofacial fueron sometidos a tratamiento quirúrgico en el HHHA de Temuco. Al Ahmed et al. (2004), en un estudio retrospectivo de 4 años, evaluaron 230 pacientes tratados por fracturas maxilofaciales de los cuales $92 \%$ eran hombres y el $8 \%$ mujeres. Nuestros resultados son similares con un $91,7 \%$ hombres y $8,3 \%$ mujeres. En el estudio de Motamedi (2003), el trauma maxilofacial afectó a los hombres en un $89 \%$ y a las mujeres en un $11 \%$. Asimismo, para Rojas et al., los pacientes tratados en el Servicio de Cirugía Maxilofacial del Hospital del Tra- 
MARTíNEZ, F.; UNIBAZO, A.; ALMEIDA, A.; ALISTER, H. J. P. \& OLATE, S. Estudio descriptivo del sistema hospitalario en relación a pacientes sometidos a tratamiento quirúrgico por trauma facial en el Hospital Hernán Henríquez Aravena de Temuco, Chile. Int. J. Odontostomat., 5(2):141-146, 2011.

bajador de Santiago de Chile, los hombres representaron $90,6 \%$, y las mujeres $9,4 \%$. Las diferentes condicionantes poblacionales llevan a que el hombre sea involucrado con mas frecuencia en eventos que determinan el trauma y las fracturas faciales.

En relación a la edad, nuestros resultados coinciden con los de Rojas et al. en Chile, Motamedi en Irán, Al Ahmed et al. en Emiratos Árabes Unidos, Brasileiro \& Passeri (2006) en Brasil, Erol et al. en Turquía, Bakardjiev \& Pechalova (2007) en Bulgaria, AlKhateeb \& Mohammad (2007) en Emiratos Árabes Unidos y Lee et al. en Korea, donde el grupo de 20 a 29 años presentó mayor cantidad de fracturas maxilofaciales. Ya en Nigeria, Adebayo et al. (2003) y en Turquía Erol et al., concentraron mayor prevalencia en sujetos de entre 30-39 años y 0-10 años respectivamente. Posiblemente las diferencias sean asociadas a la composición de la población, así como a las actividades laborales que son realizadas, donde el mayor riesgo se presenta en sujetos hombres de 20 a 40 años.

Un dato pocas veces evaluado es el asociado a las condiciones socioeconómicas de los pacientes. El $\mathrm{HHHA}$, es un establecimiento de atención pública, donde el $75,9 \%$ de nuestros pacientes poseen FONASA (sistema público de salud de Chile) como sistema de previsión. En un estudio realizado por Alvear et al. (2010) establecieron que el costo por cama día, en la especialidad de trauma, era de $\$ 331.444$ (pesos chilenos) en el Hospital de Curicó, Chile, lo que puede ser extrapolado para los pacientes de trauma facial con condicionantes similares; tiempos de hospitalización prolongados implican asumir elevados costos en los hospitales públicos; si relacionamos un menor volumen quirúrgico en el mes de febrero (probablemente debido a vacaciones del personal) y lo vinculamos al elevado costo del dia cama de un servicio de trauma, los costos son muy elevados y justifican la contratación de personal de reemplazo para la temporada de vacaciones que puedan potenciar el servicio y justifica también la implementación de un servicio que proceda con resolución rápida de los casos de trauma. En este sentido se identifica la necesidad de establecer un equipo sólido y consistente que sea capaz de intervenir en horarios diferentes a los presentes actualmente. Otra opción puede ser considerada con un servicio de urgencia que preste atención en el ámbito de la cirugía maxilofacial.

La fractura de hueso cigomático fue la más prevalente $(24,1 \%)$, sin embargo, para Motamedi en un estudio retrospectivo de 5 años en Irán, observó que de un total de 275 pacientes, la fractura más prevalente fue la fractura mandibular con un $72 \%$. Por otra parte, Erol et al., en su estudio retrospectivo de 25 años realizado en Turquía presentó $72,8 \%$ de fracturas mandibulares. Bakardjiev \& Pechalova reportan un $74 \%$ de fracturas mandibulares en individuos búlgaros así como también Al-Khateeb \& Mohammad señalaron un 70,5\% de fractura mandibular en los Emiratos Árabes Unidos. Nuestro estudio presentó la fractura mandibular en un $14,5 \%$, siendo la más prevalente la fractura de hueso cigomático con un 24,1\%. Brasileiro \& Passeri en Brasil, reportan en su estudio que la fractura mandibular $(44,2 \%)$ y cigomático $(32,5 \%)$ fueron de mayor prevalencia, similar al estudio de Maliska et al. (2009), en el mismo país, con un reporte de fractura mandibular de $54,6 \%$ y de hueso cigomático de $27,6 \%$. Coincidentemente, la fractura de mandíbula y hueso cigomático son las más prevalentes, probablemente por su posición más proyectada en el esqueleto facial.

Distintos fueron los resultados de Lee et al., quienes en un estudio retrospectivo de 4 años reportaron mayoritariamente un $42,5 \%$ de fracturas nasales, lo que contrasta con el estudio de Al Ahmed et al. con sólo un $2 \%$. Compartimos la opinión de Le et al. quienes relataron que la atención del trauma varía en diferentes instituciones de acuerdo a las preferencias del equipo tratante y de los protocolos de atención. Por otra parte, los resultados del presente estudio corresponde sólo a los sometidos a tratamiento quirúrgico; esto significa que muchos traumas de mandíbula pudieron ser tratados de forma ortopédica, aún presentando indicación de tratamiento quirúrgico, lo que puede incidir en el bajo porcentaje de fracturas mandibulares tratadas de forma quirúrgica.

El tratamiento más prevalente de nuestro estudio fue la reducción con osteosíntesis múltiple representado por un $75,2 \%$, a diferencia de los estudios de Erol et al., donde el $77,9 \%$ de sus casos los resolvieron con fijación maxilomandibular a través de arco y el $22,1 \%$ con fijación interna con placas de osteosíntesis; semejante fue el caso de Bakardjiev \& Pechalova que señalan un $78 \%$ reducción cerrada. En el estudio de Maliska et al. el 41,6\% recibió tratamiento quirúrgico, $26,5 \%$ tratamiento no quirúrgico y el $31,9 \%$ tratamiento mixto. Estas condiciones son complejas de analizar pues nuestra muestra presentó solo pacientes con tratamiento quirúrgico; por este motivo, nuevos estudios serán realizados para evaluar con detalle el tratamiento del trauma facial en estos pacientes. 
MARTíNEZ, F.; UNIBAZO, A.; ALMEIDA, A.; ALISTER, H. J. P. \& OLATE, S. Estudio descriptivo del sistema hospitalario en relación a pacientes sometidos a tratamiento quirúrgico por trauma facial en el Hospital Hernán Henríquez Aravena de Temuco, Chile. Int. J. Odontostomat., 5(2):141-146, 2011.

MARTíNEZ, F.; UNIBAZO, A.; ALMEIDA, A.; ALISTER, H. J. P. \& OLATE, S. Descriptive study of hospital system in relation to patients undergoing surgical treatment of facial trauma in the Hernan Henriquez Aravena Hospital of Temuco. Int. J. Odontostomat., 5(2):141-146, 2011.

ABSTRACT: Maxillofacial trauma constitutes a series of injuries involving bone and soft tissues, causing functional and aesthetic alterations. The aim of this study is to determine the profile of patients subject surgical treatment for maxillofacial trauma in the halls of the Regional Hospital in Temuco. From the central database of statistics Hernan Henriquez Aravena Regional Hospital we obtained a total of 145 patients with maxillofacial trauma between 2008 and 2009. In the same database the data was obtained to determine the frequency by year, ethnicity, sex, age, form of payment, month, day, service, diagnosis of fracture, soft tissue management and treatment. In this study we found 28 cases diagnosed (19.3\%), 35 fractures of zygomatic (24.1\%), 17 orbital floor fractures (11.7\%), 22 fractures of other bones of the skull $(15,2 \%), 21$ fractures of the mandible (14.5\%), 11 multiple facial fractures $(7.6 \%), 3$ fractures of the skull base $(2.1 \%)$ and 8 others $(5.5 \%)$. In conclusion we can say that zygomatic fractures is the most prevalent, and treatment performed most frequently is the reduction with multiple fixations.

KEY WORDS: surgical treatment, maxillofacial trauma, facial fractures.

\section{REFERENCIAS BIBLIOGRAFICAS}

Adebayo, E. T; Ajike, O. S. \& Adekeye, E. O. Analysis of the pattern of maxillofacial fractures in Kaduna, Nigeria. Br. J. Oral Maxillofac. Surg., 41(6):396-400, 2003.

Al Ahmed, H.; Jaber, M. A.; Abu Fanas, S. H. \& Karas, M. The pattern of maxillofacial fractures in Sharjah, United Arab Emirates: A review of 230 cases. Oral Surg. Oral Med. Oral Pathol. Oral Radiol. Endodontol., 98(2):166-70, 2004.

Al-Khateeb, T. \& Mohammad, F. Craniomaxillofacial Injuries in the United Arab Emirates: A Retrospective Study. J. Oral Maxillofac. Surg., 65(6):1094-101, 2007.

Alvear, S.; Canteros, J. \& Rodríguez P. Estudio retrospectivo de costos de tratamientos intensivos por paciente y día cama. Rev. Med. Chile, 138(5):55866, 2010.

Bakardjiev, A. \& Pechalova, P. Maxillofacial fractures in Southern Bulgaria - a retrospective study of 1706 cases. J. Craniomaxillofac. Surg., 35(3):147-50, 2007.

Erol, B.; Tanrikulu, R. \& Görgün, B. Maxillofacial Fractures. Analysis of demographic distribution and treatment in 2901 patients (25-year experience). J. Craniomaxillofac. Surg., 32(5):308-13, 2004.

Brasileiro, B. F. \& Passeri, L. A. Epidemiological analysis of maxillofacial fractures in Brazil: A 5-year prospective study. Oral Surg. Oral Med. Oral Pathol. Oral Radiol. Endod., 102(1):28-34, 2006.
Lee, J. H.; Cho, B. K. \& Park, W. J. A year retrospective study of facial fractures on Jeju, Korea. J. Craniomaxillofac. Surg., 38(3):192-6, 2010.

Le, B. T.; Holmgrem, E. P.; Holmes, J. D.; Ueeck, B. A. \& Dierks E. J. Referral patterns for the treatment of facial trauma in teaching hospitals in the United States. J. Oral Maxillofac. Surg., 61(5):557-60, 2003.

Maliska, M. C.; Lima Júnior, S. M. \& Gil, J. N. Analysis of 185 maxillofacial fractures in the state of Santa Catarina, Brazil. Braz. Oral Res., 23(3):268-74, 2009.

Medina, M.; Molina, P.; Bobadilla, L.; Zaror, R. \& Olate, $\mathrm{S}$. Fracturas maxilofaciales en individuos chilenos. Int. J. Morphol., 24(3):423-8, 2006.

Motamedi, M. H. An assessment of maxillofacial fractures: A 5-year study of 237 patients. J. Oral Maxillofac. Surg., 61(1):61-4, 2003.

Rojas, R.; Julián, G. \& Lankin J. Fracturas mandibulares: Experiencia en un hospital de trauma. Rev. Med. Chile, 130(5):537-43, 2002.

Dirección de correspondencia:

Dr. Andrés Almeida

Servicio de Cirugía Maxilofacial

Hospital Hernán Henríquez Aravena

Manuel Montt 115, Temuco,

CHILE

Email: almeida.andres@yahoo.com

Recibido : 09-06-2011

Aceptado: 11-07-2011 